\title{
Presencia de aflatoxina M1 en fórmulas lácteas infantiles comercializadas en el área metropolitana a Asunción, Paraguay
}

\section{Presence of aflatoxin M1 in infant milk formulas marketed in the metropolitan area of Asunción, Paraguay}

\author{
Andrea Alejandra Arrúa ${ }^{1,2}$ (E) Pablo David Arrua ${ }^{1}$, María Gabriela Ulke²@, Martha Yolanda

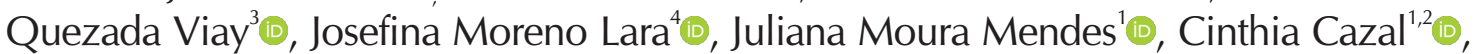

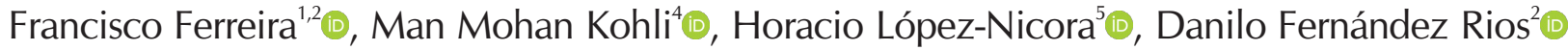 \\ ${ }^{1}$ Universidad Nacional de Asunción. Dirección General de Investigaciones Científicas y Tecnológicas. \\ Centro Multidisciplinario de Investigaciones Tecnológicas. San Lorenzo, Paraguay. \\ ${ }^{2}$ Universidad Nacional de Asunción. Facultad de Ciencias Exactas y Naturales. Departamento de \\ Biotecnología. San Lorenzo, Paraguay. \\ ${ }^{3}$ Universidad Nacional Autónoma de México. Facultad de Estudios Superiores de Postgrado. Cuautitlán \\ Izcalli, Estado de México. \\ ${ }^{4}$ Cámara Paraguaya de Exportadores y Comercializadores de Cereales y Oleaginosas. CAPECO. \\ Asunción, Paraguay. \\ ${ }^{5}$ Universidad San Carlos. Dirección de Investigación. Asunción, Paraguay.
}

\section{RESUMEN}

Introducción: La aflatoxina $\mathrm{M}_{1}\left(\mathrm{AFM}_{1}\right)$ es un metabolito tóxico derivado de la aflatoxina B. Su ingestión en lactantes se ha relacionado con retraso en el crecimiento, aumento de susceptibilidad a enfermedades infecciosas, reducción de la eficiencia en la inmunización y cirrosis. En Paraguay se recomienda lactancia exclusiva hasta los 6 meses, sin embargo se comercializan diferentes marcas de fórmulas infantiles dirigidas a lactantes menores de 6 meses. Objetivo: Detectar y cuantificar la presencia de $\mathrm{AFM}_{1}$ en fórmulas para lactantes comercializadas en el Área Metropolitana. Materiales y Métodos: Se adquirieron fórmulas fluidas $(n=18)$ y en polvo $(n=91)$ para lactantes de 0 a 12 meses de farmacias y supermercados del Área Metropolitana de Asunción y fueron analizados mediante el ensayo de inmunoafinidad ligado a enzimas (ELISA). Resultados: 9,75\% (0 a 6 meses) y $2 \%$ (6 a 12 meses) de las fórmulas lácteas en polvo y $100 \%$ de las fórmulas fluidas resultaron positivas para $\mathrm{AFM}_{1}$. La mediana de contenido de $\mathrm{AFM}_{1}$ en formulas en polvo fue de $1820 \mathrm{ng} / \mathrm{kg}$ y $510 \mathrm{ng} / \mathrm{kg}$ en las marcas A y B. En las fórmulas líquidas fue de $31,8 \mathrm{ng} / \mathrm{kg}$ y $33,6 \mathrm{ng} / \mathrm{kg}$ para las dos marcas analizadas respectivamente, $\mathrm{p}=0,0001$. Conclusiones: Se detectó $\mathrm{AFM}_{1}$ en todas las fórmulas líquidas analizadas, y en el 2 y 9,7\%

\section{ABSTRACT}

Introduction: Aflatoxin $\mathrm{M}_{1}\left(\mathrm{AFM}_{1}\right)$ is a toxic metabolite derived from aflatoxin $\mathrm{B}$. Its ingestion in infants has been related to growth retardation, increased susceptibility to infectious diseases, reduced immunization efficiency, and cirrhosis. In Paraguay, exclusive breastfeeding is recommended up to 6 months of age, however, different brands of infant formulas targeted at this age range are marketed. Objective: To detect and quantify the presence of $\mathrm{AFM}_{1}$ in infant formulas marketed in the Metropolitan Area. Materials and Methods: Fluid $(n=18)$ and powder $(n=91)$ formulas for infants aged 0 to 12 months were purchased from pharmacies and supermarkets in the Metropolitan Area of Asunción and were analyzed using the enzyme-linked immunoaffinity assay (ELISA). Results: $9.75 \%$ (0 to 6 months) and $2 \%$ ( 6 to 12 months) of the powdered milk formulas and $100 \%$ of the fluid formulas were positive for $\mathrm{AFM}_{1}$. The median content of $\mathrm{AFM}_{1}$ in powder formulas was $1820 \mathrm{ng} / \mathrm{kg}$ and $510 \mathrm{ng} / \mathrm{kg}$ in brands $\mathrm{A}$ and $\mathrm{B}$, while in liquid formulas it was $31.8 \mathrm{ng} / \mathrm{kg}$ and $33.6 \mathrm{ng} / \mathrm{kg}$ for the two brands analyzed respectively $(\mathrm{p}=0.0001)$. Conclusions: $\mathrm{AFM}_{1}$ was detected in all the liquid formulas analyzed, and in 2 and $9.7 \%$ of the

Correspondencia: Andrea Alejandra Arrúa Correo: andrea.arrua@cemit.una.py

Conflicto de interés: Los autores declaran no poseer conflicto de interés

Recibido: 16/11/2020 Aceptado: 12/02/2021

DOI: https://doi.org/10.31698/ped.48012021007

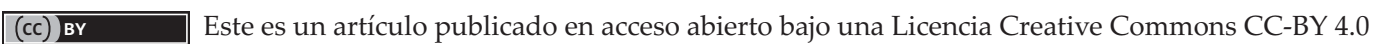


de las fórmulas en polvo de las marcas A y B respectivamente Los niveles de $\mathrm{AFM}_{1}$ fueron mayores en las fórmulas en polvo.

Palabras clave: Aflatoxinas, lactantes, alimentos formulados, intoxicación alimentaria.

\section{INTRODUCCIÓN}

Las micotoxinas son metabolitos secundarios tóxicos para humanos y animales, producidos por varias especies de hongos filamentosos. Ocasionan síndromes de síntomas diversos llamados micotoxicosis, que en casos severos pueden llevar a la muerte. Los lactantes son una de las poblaciones más vulnerables a los efectos de las micotoxinas, puesto que poseen mayor índice de metabolismo y sus sistemas inmunológicos se encuentran aún inmadu$\operatorname{ros}^{(1,2)}$. Dentro de las micotoxinas de mayor importancia se encuentran las aflatoxinas (AF), pertenecientes al Grupo 1 en la clasificación de la IARC (International Agency for Research on Cancer), lo que significa que son cancerígenas comprobadas en seres humanos. Las AF son las micotoxinas más reportadas a nivel mundial ${ }^{(1,3)}$. Además, se ha asociado la exposición a aflatoxinas al aumento de la susceptibilidad a las enfermedades infecciosas ${ }^{(4)}$, la reducción de la eficiencia en la inmunización con vacunas ${ }^{(5)} y$ cirrosis $^{(6)}$. En niños desnutridos, los riesgos para la salud asociados a las aflatoxinas se incrementan ${ }^{(7)}$.

La aflatoxina $\mathrm{M}_{1}\left(\mathrm{AFM}_{1}\right)$ es un metabolito derivado hidroxilado de la aflatoxina $\mathrm{B}$ que se produce en el tracto gastrointestinal de los mamíferos y se excreta por la leche ${ }^{(1)}$. En lactantes y niños, $\mathrm{AFM}_{1}$ se ha relacionado con retraso en el crecimiento $^{(8)}$; y si la exposición se da durante el primer año de vida, el crecimiento posterior se ve afectado.

A pesar de que el Instituto Nacional de Alimentación y Nutrición (INAN) recomienda la lactancia exclusiva hasta los 6 meses, diferentes marcas de fórmulas infantiles en polvo y fluidas se comercializan en nuestro mercado y se consumen por diversas razones asociadas a la salud del lactante como galactosemia clásica, fenilcetonuria, lactantes con muy bajo peso al nacer (menor a $1500 \mathrm{~g}$ ), recién nacidos con menos de 32 semanas de edad gestacional (muy prematuros), powder formulas of brands A and B, respectively. The levels of $\mathrm{AFM}_{1}$ were higher in the powder formulas.

Key words: Aflatoxins, infants, formulated foods, food poisoning.

recién nacidos con riesgo de hipoglicemia, entre otros problemas o bien con situaciones relacionadas a la salud del parental lactante como VIH, septicemia, herpes simplex tipo I, medicación, absceso mamario, hepatitis, mastitis, tuberculosis, adicción a drogas, entre otros ${ }^{(9)}$; por tanto, cuando el lactante debido a circunstancias especiales no puede consumir leche humana, se recurre a las fórmulas lácteas infantiles o leches adaptadas.

Considerando todo lo expuesto anteriormente y que en el país no existen datos acerca de la presencia de aflatoxinas en fórmulas para lactantes, el objetivo de este trabajo fue detectar y cuantificar la presencia de $\mathrm{AFM}_{1}$ en fórmulas para lactantes comercializadas en el Área Metropolitana de Paraguay y estimar el riesgo de ingesta de estos productos.

\section{MATERIALES Y MÉTODOS}

Este trabajo se refiere a un análisis realizado por el Centro Multidisciplinario de Investigaciones Tecnológicas, Dirección General de Investigación Científica y Tecnológica (CEMIT-DGICT) y la Facultad de Ciencias Exactas y Naturales (FACEN), ambas unidades pertenecientes a la Universidad Nacional de Asunción (UNA), y corresponde al análisis de fórmulas en polvo y fluidas para lactantes de 0 a 12 meses de edad de acuerdo con el etiquetado.

Se realizó un estudio observacional descriptivo de corte transversal con muestreo aleatorio simple. La colecta de los productos se realizó durante el mes de enero de 2018, de farmacias y supermercados de Asunción y Área Metropolitana (San Lorenzo, Luque, Fernando de la Mora y Mariano Roque Alonso), que comprende aproximadamente el 43\% de la población del país. Se excluyeron aquellos que se encontraban alterados o con fecha vencida. 
La unidad de análisis consistió en empaques tipo caja de 200, 400 y 800 g y latas de 400 y 800 g de fórmulas comerciales en polvo para lactantes de $0 \mathrm{a}$ 12 meses de edad de acuerdo con el etiquetado. Todos los productos analizados fueron importados de los siguientes países: fórmulas lácteas en polvo: Argentina (78) y México (14); fórmulas lácteas fluidas: Argentina (18).

Los productos fueron transportados al Laboratorio de Biotecnología del CEMIT-DGICT-UNA y almacenados en condiciones adecuadas para el análisis, de acuerdo con el Código de Prácticas de Higiene para la Leche y los Productos Lácteos de la FAO (Organización de las Naciones Unidas para la Alimentación y la Agricultura) ${ }^{(10)}$.

\section{Extracción de micotoxinas}

\section{Fórmulas en polvo}

La extracción de micotoxinas se realizó de acuerdo con las instrucciones del fabricante, siguiendo el siguiente procedimiento: Para preparar muestras para la prueba ELISA, se colocaron $10 \mathrm{~g}$ de fórmula láctea en polvo en un matraz Erlenmeyer con tapa y se sugiere, 10 gramos de fórmula se disolvieron en $100 \mathrm{~mL}$ de agua desionizada esterilizada a $50^{\circ} \mathrm{C}$. Se repite $50^{\circ} \mathrm{C}$, homogeneizando mediante agitación con un agitador magnético. Posteriormente, se tomaron mediante una pipeta $5 \mathrm{~mL}$ de esta preparación, se transfirieron a tubos de ensayo y se incubaron a $4{ }^{\circ} \mathrm{C}$ durante 30 minutos. Posteriormente, las muestras se centrifugaron a $3000 \mathrm{~g}$ durante 10 minutos y se tomaron $0,4 \mathrm{~mL}$ del suero por debajo de la capa de grasa. El suero colectado se mezcló con $0,1 \mathrm{~mL}$ de metanol al $100 \%{ }^{(11)}$.

\section{Fórmulas fluidas}

Se transfirieron mediante una pipeta $5 \mathrm{~mL}$ de la fórmula láctea fluida a un tubo de ensayo de $15 \mathrm{~mL}$ y se incubaron a $4{ }^{\circ} \mathrm{C}$ durante 30 minutos. Posteriormente, las muestras se centrifugaron a 3000 $g$ durante 10 minutos, se tomaron $0,4 \mathrm{~mL}$ del suero debajo de la capa de grasa. El suero colectado se mezcló con $0,1 \mathrm{~mL}$ de metanol al $100 \%{ }^{(11)}$.

\section{Determinación de micotoxinas}

Para la determinación de $\mathrm{AFM}_{1}$ se utilizó el kit AgraQuant ${ }^{\circledR}$ ELISA Aflatoxin $\mathrm{M}_{1}$ Sensitive 25 - 500 ppt. Para el análisis se consideraron como muestras positivas aquellas que presentaron valores de $\mathrm{AFM}_{1}$ a partir del límite de detección de $200 \mathrm{ng} / \mathrm{kg}$ para las fórmulas en polvo y de $25 \mathrm{ng} / \mathrm{kg}$ para las fórmulas fluidas según la metodología utilizada ${ }^{(11)}$. Se determinaron los valores máximos, mínimos, la mediana y la media de $\mathrm{AFM}_{1}$ presente en las fórmulas lácteas infantiles analizadas.

\section{Análisis de los datos}

La comparación de las medias de la concentración de $\mathrm{AFM}_{1}$ fue realizada con el paquete estadístico Infostat ${ }^{\circledR}{ }^{(12)}$ utilizando el análisis de varianza no paramétrica (ANOVA) con un intervalo de confianza del $95 \%$ a través de la prueba de Tukey, considerando un error alfa del $5 \%$.

\section{Cuestiones éticas}

El estudio realizado no incluyó animales ni seres humanos. Todos los productos fueron adquiridos en farmacias y supermercados de Asunción y Área Metropolitana (San Lorenzo, Luque, Fernando de la Mora y Mariano Roque Alonso) donde se venden de manera irrestricta.

\section{RESULTADOS}

Fueron incluidas 109 muestras entre fórmulas líquidas y en polvo (Tabla 1).

Tabla1. Muestras de fórmulas infantiles N=109

\begin{tabular}{lcc}
\hline Producto & Edad en meses & Número de muestras \\
\hline Fórmula infantil en polvo & $0-6$ & 42 \\
Fórmula infantil en polvo & $6-12$ & 49 \\
Fórmula infantil fluida & $0-6$ & 9 \\
Fórmula infantil fluida & $6-12$ & 9 \\
\hline Total de muestras & & $\mathbf{1 0 9}$ \\
\hline
\end{tabular}




\section{Contenido de AFM1 en las muestras}

\section{Fórmulas lácteas en polvo}

En las fórmulas lácteas en polvo para lactantes de $0 \mathrm{a}$ 6 meses, cuatro muestras $(9,75 \%)$, todas de la misma marca y de dos diferentes lotes, presentaron valores positivos de $\mathrm{AFM}_{1}$. En relación con las fórmulas lácteas en polvo destinadas a niños de 6 a 12 meses de edad, una de las muestras $(2 \%)$ presentó una concentración de $510 \mathrm{ng} / \mathrm{kg}$. No se observaron diferencias significativas entre las medias de las fórmulas en polvo destinadas a lactantes de 0 a 6 meses y de 6 a 12 meses estudiadas ( $p=0.26$ ) (Tabla 2).

\section{Fórmulas lácteas fluidas}

El 100\% de las fórmulas fluidas analizadas presentó contaminación con $\mathrm{AFM}_{1}$. No se observaron diferencias significativas entre la mediana de las fórmulas fluidas analizadas $(\mathrm{p}=0,60)$. Los contenidos de $\mathrm{AFM}_{1}$ en las formulas en polvo para lactantes de 0 a 6 meses y de 6 a 12 meses fueron superiores a los detectados en los productos líquidos (Tabla 2).

Tabla 2. Contenido de AFM1 en formulas infantiles en polvo (ng/kg)

\begin{tabular}{lcccccc}
\hline Tipo de formula láctea & $\begin{array}{c}\text { Edad } \\
\text { recomendada }\end{array}$ & Marca & $\begin{array}{c}\text { Mínimo nivel } \\
\text { detectado }\end{array}$ & $\begin{array}{c}\text { Máximo nivel } \\
\text { detectado }\end{array}$ & Mediana & Valor de $\boldsymbol{p}$ \\
\hline $\begin{array}{l}\text { Formula láctea } \\
\text { polvo* }\end{array}$ & $\begin{array}{c}0-6 \text { meses } \\
\mathrm{nc}=4\end{array}$ & $\mathrm{~A}$ & 610 & 2100 & 1820 & 0.26 \\
\hline $\begin{array}{l}6-12 \text { meses } \\
\mathrm{nc}=1\end{array}$ & $\mathrm{~A}$ & 510 & 510 & 510 & $p=0,0001$ \\
\hline $\begin{array}{l}\text { Formula láctea } \\
\text { fluida** }\end{array}$ & $\begin{array}{c}\mathrm{nc} \text { meses } \\
6 \mathrm{a} 12 \text { meses } \\
\mathrm{nc}=9\end{array}$ & $\mathrm{~B}$ & 22,6 & 38,9 & $31,8 \mathrm{abb}$ & 0.60 \\
& $\mathrm{~B}$ & 25,6 & 45,5 & $33,6 \mathrm{bb}$ & $p=0,0001$ \\
\hline
\end{tabular}

* $\mathrm{DL}=252 \mathrm{ng} / \mathrm{kg}$ para fórmulas lácteas en polvo $* * \mathrm{DL}=18 \mathrm{ng} / \mathrm{kg}$ para fórmulas lácteas fluidas. Valor de $p$ calculado mediante uso de paquete estadístico Infostat.

Las marcadas diferencias entre los porcentajes de muestras positivas en las fórmulas lácteas fluidas y las fórmulas lácteas en polvo estudiadas, pueden deberse principalmente a los límites de detección (DL) de la técnica utilizada, de DL $=252 \mathrm{ng} / \mathrm{kg}$ para fórmulas lácteas en polvo $\mathrm{DL}=18 \mathrm{ng} / \mathrm{kg}$ para fórmulas lácteas fluidas. Es destacable que el límite de detección para los productos fluidos es mucho menor al de los productos en polvo.

\section{DISCUSIÓN}

En el presente estudio, el análisis de fórmulas lácteas comerciales en polvo y fluidas reveló la presencia de $\mathrm{AFM}_{1}$ en niveles de superiores al límite establecido por la Comisión Europea en media (de $25 \mathrm{ng} / \mathrm{kg})^{(13)}$.
Paraguay no cuenta con ninguna reglamentación sobre el límite de $\mathrm{AFM}_{1}$ en las fórmulas comerciales; sin embargo, la norma del MERCOSUR indica como límite de $\mathrm{AFM}_{1}$ para leche fluida $500 \mathrm{ng} / \mathrm{kg}$, y para leche en polvo $5000 \mathrm{ng} / \mathrm{kg}^{(14)}$.

La presencia de $\mathrm{AFM}_{1}$ como contaminante en fórmula láctea comercial ha sido reportada en diferentes países alrededor del mundo. En Líbano, en 42 marcas de fórmulas lácteas analizadas mediante test de ELISA, la concentración media de $\mathrm{AFM}_{1}$ fue de 20,1 $\pm 1,3 \mathrm{ng} / \mathrm{kg}$, el nivel de contaminación osciló entre no detectado y $48,1 \mathrm{ng} / \mathrm{L}$; $31 \%$ de las marcas presentaron nivel promedio por encima del límite de la Comisión Europea ${ }^{(15)}$. 
Un análisis de presencia de $\mathrm{AFM}_{1}$ en fórmulas para lactantes y marcas de leche disponibles en los mercados de Pakistán realizado en 2017 halló que el $30,76 \%$ de las muestras de fórmulas para lactantes excedieron el límite de la Comisión Europea ${ }^{(16)}$. En ambos estudios mencionados anteriormente, el porcentaje de muestras contaminadas fue ampliamente superior al nivel de contaminación de los productos comercializados en el Área Metropolitana de Asunción detectado por este estudio, que varió entre 2 y $9,75 \%$.

En el sur de Brasil, se analizaron 16 marcas de fórmula en polvo para lactantes y se detectó $\mathrm{AFM}_{1}$ en $43,8 \%(n=7)$ de las muestras, con una media de 0,011 $\mathrm{ng} / \mathrm{g}$, inferior a lo establecido por las directrices brasileñas $(5 \mathrm{ng} / \mathrm{g})$; tres $(18,75 \%)$ de las muestras analizadas excedieron el límite establecido por la Comisión Europea ${ }^{(13,17)}$.

Más recientemente, se ha descubierto una alta presencia de $\mathrm{AFM}_{1}$ en fórmulas infantiles distribuidas en el área metropolitana de Monterrey (México). De 55 muestras evaluadas, el 20\% presentó un rango de 40-450 $\mathrm{ng} / \mathrm{kg}$, por encima del límite de la Comisión Europea ${ }^{(18)}$.

La estimación de la ingesta diaria (EID) fue calculada en un estudio previo realizado en Monterrey, México se reportaron valores de EID en un rango de 4,39-2,55 y 2,55-1,56 para bebés de 6 a 12 meses ${ }^{(18)}$. En Líbano, la ingesta de $\mathrm{AFM}_{1}$ a través de fórmulas lácteas infantiles se estimó en $0,47 \mathrm{ng} / \mathrm{kg}$ pc/día ${ }^{(15)}$. En Paraguay no existen estadísticas acerca del consumo de fórmulas lácteas (marcas, cantidades, ingesta diaria) y el Ministerio de Salud recomienda la lactancia materna exclusiva hasta los 6 meses de edad, sin embargo, en el mercado nacional se comercializan en farmacias, supermercados e inclusive en las calles fórmulas lácteas para lactantes y queda patente la necesidad de datos fidedignos que permitan calcular valores reales de EID para nuestro país.

Teniendo en cuenta la carcinogenicidad de las aflatoxinas, es deseable que el nivel presente en los alimentos sea cero, pero en la realidad esto es imposible puesto que los hongos productores de micotoxinas son ubicuos y universales, y contaminan una gran variedad de sustratos alimenticios en todas las etapas de la cadena

productiva, y por tanto su control se hace extremadamente difícil, por tanto es necesario que los contenidos de $\mathrm{AFM}_{1}$ sean lo más cercanos posibles al cero, siguiendo el principio ALARA ( $A s$ Low As Reasonably Achievable), "tan bajo como fuera posible", por lo cual establecer legislaciones que limiten los contenidos de esta micotoxina en fórmulas lácteas comerciales y realizar controles y monitoreos aleatorios de fórmulas lácteas es prioritario y urgente.

En nuestro estudio, a pesar de que el número de muestras contaminadas es bajo en comparación con trabajos realizados en otros países en matrices similares, los contenidos de $\mathrm{AFM}_{1}$ son extremadamente altos. El bajo porcentaje de muestras positivas en el estudio realizado en Paraguay podría deberse a que el límite de detección de la técnica para las fórmulas en polvo es de 200 $\mathrm{ng} / \mathrm{kg}$, lo que indica la necesidad de realizar estudios con técnicas más sensibles, sobre todo cuando se trata de alimentos destinados a poblaciones vulnerables, como los lactantes. Con relación a las fórmulas fluidas, los valores de $\mathrm{AFM}_{1}$ fueron bajos en comparación con las fórmulas en polvo, sin embargo, aun así, superaron el valor de la normativa europea. Este es un llamado de atención para las autoridades de salud, ya que indica la urgente necesidad de realizar monitoreos de micotoxinas de productos destinados al consumo de lactantes ya que dada la toxicidad de $\mathrm{AFM}_{1}$ y el consumo diario de leche por parte de los lactantes, la exposición dietética a largo plazo puede representar un riesgo significativo.

La prueba ELISA es un indicador adecuado de la presencia de micotoxinas en diferentes matrices alimentarias como análisis preliminar ${ }^{\left({ }^{(1)}\right)}$ y una opción válida para determinar la contaminación por micotoxinas cuando no se dispone de técnicas más sensibles como UPLC / HPLC ${ }^{(20)}$. Uno de los principales obstáculos en Paraguay es la falta de laboratorios que realicen análisis extremadamente precisos, por lo cual la prueba ELISA se convierte en la mejor alternativa para su rápida, económica y efectiva determinación de micotoxinas en alimentos. Recientemente fue publicado en Paraguay un estudio acerca de la presencia de micotoxinas en preparados y colados comerciales para lactantes, en 
el cual, mediante el uso del test de ELISA, se detectó la contaminación de estos productos con aflatoxinas, ocratoxinas, toxina T-2 y deoxinivalenol ${ }^{(2)}$. Debido a la presencia de $\mathrm{AFM}_{1}$ en fórmulas lácteas infantiles comprobada por los resultados de este estudio, es necesaria la implementación de técnicas más sensibles para la detección de micotoxinas en fórmulas lácteas y alimentos para lactantes ya que la sensibilidad del test de ELISA podría resultar una limitante.

Los resultados de este estudio muestran la presencia de $\mathrm{AFM}_{1}$ en fórmula láctea en polvo y fluida comercializada en Paraguay, y resaltan la necesidad de que las autoridades realicen monitoreos aleatorios y regulares que aseguren la inocuidad de estos productos, y de que los productores e industrias apliquen medidas que prevengan $y$

\section{REFERENCIAS}

1. IARC. A review of human carcinogens. In: IARC Working Group on the Evaluation of Carcinogenic Risks to Humans, editor. A review of human carcinogens Part F: Chemical agents and related occupations. Lyon, France: International Agency for Research on Cancer, World Health Organization; 2009. p. 225-39.

2. Arrúa AA, Arrúa PD, Mendes JM, Ferreira FP, Fernández Ríos D, Ulke MG, et al. Presencia de micotoxinas en preparados y colados comerciales para lactantes en el área metropolitana de Asunción, Paraguay. Pediatr (Asunción) [Internet]. 2019 [cited 2020 Mar 28];46(2):97-102. Available f $\quad r \quad$ o $\quad$ m https://revistaspp.org/index.php/pediatria/article/view/497

3. Patriarca A, Fernández Pinto V. Prevalence of mycotoxins in foods and decontamination. Curr Opin Food Sci [Internet]. 2017[cited 2019 Mar 28];14:50-60. Available from: https://linkinghub.elsevier.com/retrieve/pii/S22147993173 00152

4. Turner PC, Mendy M, Whittle H, Fortuin M, Hall AJ, Wild CP. Hepatitis B infection and aflatoxin biomarker levels in Gambian children. Trop Med Int Heal [Internet]. [cited 2019 Mar 28]Available from: http://doi.wiley.com/ 10.1046/j.1365-3156.2000.00664.x reduzcan la contaminación por micotoxinas de las materias primas utilizadas en la producción de alimentos infantiles con medidas para controlar los niveles de micotoxinas a través de sistemas de gestión de calidad e inocuidad de alimentos ${ }^{(21)}$.

\section{CONCLUSIONES}

Los resultados del presente estudio han demostrado la presencia de $\mathrm{AFM}_{1}$ en fórmulas lácteas infantiles distribuidas en el Área Metropolitana de Asunción (Paraguay).

El $100 \%$ de las muestras positivas presentaron contenidos de $\mathrm{AFM}_{1}$ en un rango de entre 510 a 1820 $\mathrm{ng} / \mathrm{kg}$ en fórmula láctea en polvo y 22,6 a 45,5 ng/kg en fórmula láctea fluida.
5. Raiola A, Tenore GC, Manyes L, Meca G, Ritieni A. Risk analysis of main mycotoxins occurring in food for children: An overview. Food Chem Toxicol [Internet]. 2015[cited 2019 Mar 28];84:169-80. Available from: http://dx.doi.org/10.1016/j.fct.2015.08.023

6. Ramalho LNZ, Porta LD, Rosim RE, Petta T, Augusto MJ, Silva DM, et al. Aflatoxin B1 residues in human livers and their relationship with markers of hepatic carcinogenesis in São Paulo, Brazil. Toxicol Reports [Internet]. 2018[cited 2019 Dec 18];5:777-84. Available from:https://doi.org/10.1016/j.toxrep.2018.07.005

7. McMillan A, Renaud JB, Burgess KMN, Orimadegun $\mathrm{AE}$, Akinyinka OO, Allen SJ, et al. Aflatoxin exposure in Nigerian children with severe acute malnutrition. Food Chem Toxicol [Internet]. 2018 [cited 2019 Dec 18];111:356-62. Available from: https://doi.org/10.1016/ j.fct.2017.11.030

8. Turner PC, Collinson AC, Cheung YB, Gong Y, Hall AJ, Prentice AM, et al. Aflatoxin exposure in utero causes growth faltering in Gambian infants. Int J Epidemiol [Internet]. 2007[cited 2019 Dec 11];36(5):1119-25. Available from: https://academic.oup.com/ije/articlelookup/doi/10.1093/ije/dym122 
9. WHO. Infant and young child feeding. Model Chapter for textbooks for medical students and allied health professionals [Internet]. World Health Organization. Geneva: WHO Press, World Health Organization; 2009 [cited 2020 Sep 9]. 112 p. Available from: https://www.wh o.int/news-room/fact-sheets/detail/infant-and-youngchild-feeding

10. FAO. Productos que se mantienen estables a temperatura ambiente. In: CAC/RCP 57, editor. Código de prácticas de Higiene para la leche y los productos lácteos. Roma: Organización de las Naciones Unidas para la Alimentación y la Agricultura; 2004. p. 41.

11. ROMER. AgraQuant ${ }^{\circledR}$ Aflatoxin M1 Sensitive 25/500. Romer Labs. Romer Labs; 2010. 6-9 p.

12. Di Rienzo JA, Casanoves F, Balzarini MG, Gonzalez L, Tablada M, Robledo y C. InfoStat [Internet]. Grupo InfoStat. Córdoba, Argentina: Universidad Nacional de Córdoba; 2011[cited 2020 Sep 28]. Available from: http://www.infostat.com.ar

13. Commission Regulation. Commission Regulation (EC) No 1881/2006 of 19 December 2006 setting maximum levels for certain contaminants in foodstuffs (Text with EEA relevance) [Internet]. EUR-Lex. 2006 [cited 2020 Sep 29]. p. 558-577. Available from: https://eur-lex.europa.eu/eli/ reg/2006/1881/oj

14. MERCOSUR. Reglamento técnico sobre límites máximos de aflatoxinas [Internet]. Resolución. Resolución N 56/94. Brasilia, Brazil: Mercado Común del Sur (MERCOSUR): Grupo Mercado Común (GMC); 1994 [cited 2020 Sep 12]. p. 6. Available from: https://www.impo.com.uy/bases/ decretos-reglamento/155-2006/2

15. Elaridi J, Dimassi H, Hassan H. Aflatoxin M1 and ochratoxin A in baby formulae marketed in Lebanon: Occurrence and safety evaluation. Food Control [Internet]. 2019[cited 2020 Sep 28];106(June):106680. Available from: https://linkinghub.elsevier.com/retrieve/ pii/S0956713519302610
16. Akhtar S, Shahzad MA, Yoo S-H, Ismail A, Hameed A, Ismail $\mathrm{T}$, et al. Determination of Aflatoxin M 1 and Heavy Metals in Infant Formula Milk Brands Available in Pakistani Markets. Korean J Food Sci Anim Resour [Internet]. 2017[cited 2020 Sep 28];37(1):79-86. Available from: http://koreascience.or.kr/journal/view.jsp?kj=CS SPBQ\&py=2017\&vnc $=v 37 n 1 \& s p=79$

17. Ishikawa A, Takabayashi-Yamashita C, Ono E, Bagatin A, Rigobello F, Kawamura O, et al. Exposure Assessment of Infants to Aflatoxin M1 through Consumption of Breast Milk and Infant Powdered Milk in Brazil. Toxins (Basel) [Internet]. 2016 [cited 2020 Sep 28];8(9):246. Available from: http://www.mdpi.com/2072-6651/8/9/246

18. Quevedo-Garza PA, Amador-Espejo GG, Salas-García R, Ramos-Peña EG, Trujillo A-J. Aflatoxin M1 Determination in Infant Formulae Distributed in Monterrey, Mexico. Toxins (Basel) [Internet]. 2020[cited 2021 Jan 28];12(2):100. Available from: https://www.mdpi.com/2072-6651/12/2/100

19. Dong A, Liu S, Bo F. Study on comparison of the different method to detect concentration of mycotoxins. China Feed [Internet]. 2017[cited 2020 Sep 28];(4):5. Available from: http://en.cnki.com.cn/Article_en/ CJFDTotal-SLGZ201704005.htm

20. Gummadidala PM, Omebeyinje MH, Burch JA, Chakraborty P, Biswas PK, Banerjee K, et al. Complementary feeding may pose a risk of simultaneous exposures to aflatoxin M1 and deoxynivalenol in Indian infants and toddlers: Lessons from a mini-survey of food samples obtained from Kolkata, India. Food Chem Toxicol [Internet]. 2019[cited 2020 Sep 28];123:9-15. Available from: https://linkinghub.elsevier.com/retrieve/pii/ S0278691518307294

21. Herrera M, Bervis N, Carramiñana JJ, Juan T, Herrera A, Ariño A, et al. Occurrence and Exposure Assessment of Aflatoxins and Deoxynivalenol in Cereal-Based Baby Foods for Infants. Toxins (Basel) [Internet]. 2019 Mar 5 [cited 2019 Mar 28];11(3):150. Available from: https://www.mdpi.com/2072-6651/11/3/150 